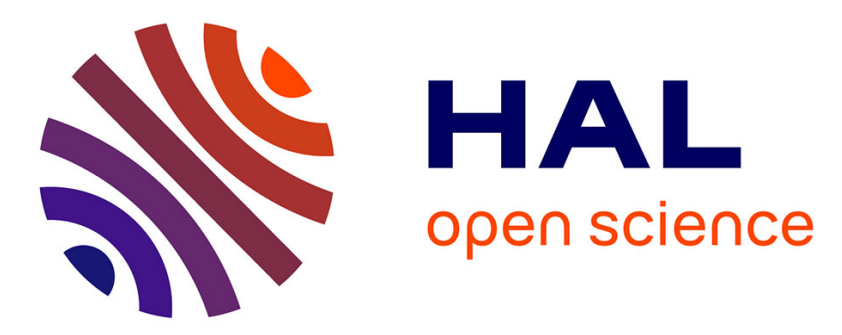

\title{
Modulated magnetic structures of the antiferromagnetic hydride CeRuSiH
}

\author{
Sophie Tencé, Gilles André, Etienne Gaudin, Bernard Chevalier
}

\section{To cite this version:}

Sophie Tencé, Gilles André, Etienne Gaudin, Bernard Chevalier. Modulated magnetic structures of the antiferromagnetic hydride CeRuSiH. Journal of Physics: Condensed Matter, 2008, 20 (25), 255239 (6 p.). 10.1088/0953-8984/20/25/255239 . cea-00285472

\section{HAL Id: cea-00285472 https://hal-cea.archives-ouvertes.fr/cea-00285472}

Submitted on 5 Jun 2008

HAL is a multi-disciplinary open access archive for the deposit and dissemination of scientific research documents, whether they are published or not. The documents may come from teaching and research institutions in France or abroad, or from public or private research centers.
L'archive ouverte pluridisciplinaire HAL, est destinée au dépôt et à la diffusion de documents scientifiques de niveau recherche, publiés ou non, émanant des établissements d'enseignement et de recherche français ou étrangers, des laboratoires publics ou privés. 


\section{Modulated magnetic structures of the antiferromagnetic hydride CeRuSiH}

\section{Tencé S., André G., Gaudin E., Chevalier B.}

\section{Introduction}

Recently, it was established that the ternary compounds CeMX ( $M=$ transition metal and $X=S i$ or $\mathrm{Ge}$ ) crystallizing in the tetragonal CeFeSi-type structure absorb hydrogen at moderate temperature $(523 \mathrm{~K})$ and produce stable hydrides CeMXH in ambient conditions [1-6]. The investigation of the structural properties of these hydrides reveals that the hydrogenation: (i) allows us to preserve the initial crystal symmetry of the intermetallics ( $P 4 / \mathrm{nmm}$ space group); (ii) induces an anisotropic evolution of the unit cell parameters since a decreases weakly whereas $C$ increases strongly, therefore the unit cell volume increases and finally (iii) allows us to insert the $\mathrm{H}$-atoms inside the $\left[\mathrm{Ce}_{4}\right]$ pseudotetrahedral sites [3]. It is noteworthy that the crystal structure of the hydrides CeMXH can be compared to those of phosphide oxides CeMPO ( $\mathrm{M}=\mathrm{Fe}$, Co or Ru) where O-atoms occupy also the $\left[\mathrm{Ce}_{4}\right]$ tetrahedral arrangements $[7,8]$. Recent work reports that CeRuPO is a rare example of a ferromagnetic Kondo lattice [8]. The hydrogenation of the ternary compounds adopting the tetragonal CeFeSi-type structure allows us to study the influence on their physical properties of the competition between two effects: (i) the increase of the unit cell volume linked to the $\mathrm{H}$-insertion and (ii) the occurrence of the $\mathrm{Ce}-\mathrm{H}$ chemical bonding. The former considered as an application of 'negative' pressure on the intermetallics, tends to stabilize the trivalent cerium by decreasing the coupling constant $J_{\mathrm{cf}}$ between $4 \mathrm{f}(\mathrm{Ce})$ and conduction electrons. Jcf governs the competition, described by the Doniach phase diagram [9], between the indirect magnetic Ruderman-Kittel-Kasuya-Yoshida (RKKY) interaction and the demagnetizing Kondo interaction. For instance, the reduction of Jcf by hydrogenation favours, for CeMX compounds strongly influenced by the Kondo interaction, the occurrence or the increase of magnetic ordering for cerium $[10,11]$. In contrast, the second effect can lead to the demagnetization of cerium if the $\mathrm{Ce}-\mathrm{H}$ bonding is strong as evidenced by investigation of the hydrides $\mathrm{CeCoSiH}$ and CeCoGeH by ${ }^{1} \mathrm{H}$ nuclear magnetic resonance (NMR) [5]. The strong chemical shift (150 ppm) determined for these hydrides suggests a transfer of some density of electron spin from Ce to proton via orbital overlap. The competition between the two effects resulting from the hydrogenation of CeMX compounds (CeFeSi-type structure) induces interesting magnetic transitions as antiferromagnetic ordering $\rightarrow$ spin fluctuation behaviour observed during the hydrogenation of CeCoSi and CeCoGe $[1,2,4,5]$ or from a non-magnetic heavy-fermion system (CeRuSi) to an antiferromagnet (CeRuSiH) [6]. This last hydride exhibits a complex magnetic phase diagram showing two antiferromagnetic transitions at $T_{\mathrm{N} 1}=7.5(2) \mathrm{K}$ and $T_{\mathrm{N} 2}=3.1(2) \mathrm{K}$ evidenced by 
magnetization and specific heat measurements. Furthermore, a metamagnetic double transition was observed at low temperature [6]. This diagram exhibits many similarities with that reported for $\mathrm{CeRu}_{2}\left(\mathrm{Si}_{1-} x \mathrm{Ge}_{x}\right)_{2}$ and $\left(\mathrm{Ce}_{1-x} \mathrm{La}_{x}\right) \mathrm{Ru}_{2} \mathrm{Si}_{2}$ systems where an antiferromagnetic ordering is induced in the non-magnetic heavy-fermion $\mathrm{CeRu}_{2} \mathrm{Si}_{2}$ by slightly expanding its lattice through the substitution of a few percent of germanium or lanthanum for silicon or cerium [12-16]. In this context, it was useful to perform neutron powder diffraction experiments on hydride CeRuSiH in order to determine its crystal and magnetic structures. In this paper, we present these structures and their evolution with the temperature. Moreover the investigation is discussed in relation to the results previously obtained on both compounds REMSi and REMGe (RE = rare earth) adopting the same tetragonal CeFeSi-type structure and on the substituted compounds $\left(\mathrm{Ce}_{1-x} \mathrm{La}_{x}\right) \mathrm{Ru}_{2} \mathrm{Si}_{2}$ and $\mathrm{CeRu}_{2}\left(\mathrm{Si}_{1-x} \mathrm{Ge}_{x}\right)_{2}$.

\section{Experimental procedures}

The CeRuSiH sample used for this study was synthesized and characterized as described previously in [6]. This hydride is stable in air and its $\mathrm{H}$-content is not affected by exposure under vacuum for temperatures smaller than $300 \mathrm{~K}$. Neutron powder diffraction experiments were performed at the Orph' ee reactor (CEA/Saclay, France) on the twoaxis diffractometers G4.1 $(\lambda=2.4226 \AA ; 800$ cellposition-sensitive detector) and 3T2 (high resolution powder diffractometer; $\lambda=1.2262 \AA$ ). Several patterns were recorded between 1.5 and $10 \mathrm{~K}$ (namely above and below the $T_{\mathrm{N} 1}$ ordering temperature) on G4.1 and one pattern at room temperature on 3T2. The data were analysed using the Rietveld profile method by means of the Fullprof program [17], with neutron-scattering lengths and Ce3+ magnetic form factor taken respectively from [18] and [19]. The Ce3+ magnetic form factor taken for cerium is justified because it corresponds to the Ce valency found in hydride CeRuSiH according to magnetic susceptibility measurements [6].

\section{Results and discussion}

\subsection{Crystal structure}

Analysis of the neutron diffraction pattern of CeRuSiH recorded at room temperature (figure 1) confirms that this hydride adopts as the parent ternary silicide CeRuSi the tetragonal CeFeSi-type structure ( $P 4 / \mathrm{nmm}$ space group). No trace of any impurity phase was detected in the pattern. The high background value is due to the high incoherent scattering cross-section of the hydrogen. The unit cell parameters $a=4.1777(1) \AA$ and $c=7.5072(1) \AA$ are in agreement with those reported earlier using $x$-ray diffraction on single crystals [6]. The results of the Rietveld profile refinement (positional and isotropic thermal parameters) are collected in table 1. All these parameters are in agreement with those reported in [6] where the crystal structure was described in detail. In particular, we confirm that $\mathrm{H}$-insertion induces an anisotropic expansion of the unit cell and affects strongly the Si atomic position. We also note a preferential orientation effect along the c-axis which has been taken into account in the final refinement. The best refinement at room temperature 
leads to reliability factors $R_{\mathrm{wp}}=8.7 \%$ and $R_{\mathrm{B}}=4.9 \%$. Moreover, several neutron diffraction patterns were recorded for CeRuSiH on G4.1 at low temperatures, between 1.5 and $10 \mathrm{~K}$. At $10 \mathrm{~K}$, above the $T_{\mathrm{N} 1}$ magnetic transition temperature, only nuclear diffraction peaks are distinguished. The unit cell parameters at $10 \mathrm{~K}$ are $a=4.1758(1) \AA$ and $c=7.4884(4) \AA$, values slightly smaller than those reported for room temperature. The best refinement at $10 \mathrm{~K}$ leads to a reliability factor of $R_{\mathrm{b}}=$ $3.4 \%$. According to our refinements, the $\mathrm{H}$-atoms occupy unambiguously the $\left[\mathrm{Ce}_{4}\right]$ tetrahedral sites (1/4 3/4 1/2) empty in CeRuSi with an occupation number of 1.03(4). The precision of the hydrogen content is quite high because the nuclear intensities are very sensitive to the H occupancy. This last parameter, refined from 3T2 data, suggests that the site [ $\left.\mathrm{Ce}_{4}\right]$ is totally occupied by hydrogen which is in fair agreement with the amount of $\mathrm{H}$ inserted in CeRuSi determined volumetrically by monitoring pressure changes in a calibrated volume during the hydride synthesis (1.0(1) per CeRuSi formula) [6]. The H-position in CeRuSiH is the same as the one of D observed in the deuteride CeCoGeD [3]. In consequence, if we calculate at room temperature the interatomic distance between $\mathrm{Ce}$ - and $\mathrm{H}$-atoms, we obtain $d_{\mathrm{Ce}-\mathrm{H}}=2.440 \AA$, which is higher than the ones reported for the hydrides CeCoSiH $\left(d_{\mathrm{Ce}-\mathrm{H}}=2.391 \AA\right)$ [2] and CeCoGeH $\left(d_{\mathrm{Ce}-\mathrm{H}}=2.410 \AA\right)$ [1]. This result suggests that the chemical bonding between $\mathrm{Ce}$ and $\mathrm{H}$ in CeRuSiH is weaker than that existing in other isomorphous hydrides based on cobalt. That could explain why in CeRuSiH the unit cell expansion prevails on the chemical effect of the $\mathrm{Ce}-\mathrm{H}$ bond and thus why the magnetic ordering appears. This was recently confirmed by electronic structure calculations using the densityfunctional-theory (DFT) [20]: the $4 f(C e)$-states for the hydride appear much localized at the Fermi level $\left(E_{\mathrm{F}}\right)$ and an antiferromagnetic structure with $\sim 1.9 \mu_{\mathrm{B}}$ as Ce magnetic moment was proposed for the CeRuSiH ground state.

\subsection{Magnetic structure}

Below the $\mathrm{N}^{\prime}$ eel temperature $T_{\mathrm{N} 1}=7.5 \mathrm{~K}$, additional peaks appear in the neutron diffraction patterns: they are characteristic of an antiferromagnetic ordering, in agreement with the magnetic measurements [6]. At $1.5 \mathrm{~K}$, these peaks can be indexed on the basis of a magnetic cell twice as large as the nuclear one along the $c$-axis and almost three times larger along the $a$ - and $b$-axes (i.e. with a propagation vector $\mathbf{k}=\left(k_{x} \approx 1 / 3, k_{x}, 1 / 2\right)$ in reduced units $)$. More precisely, the refined $k x$ value is $0.3328(1)$ at $1.5 \mathrm{~K}$. This refined value seems to indicate that the magnetic structure is not exactly commensurate. Nevertheless the discrepancy from the exact commensurate value $1 / 3$ is tiny and as the estimated standard deviations do not take into account systematic errors, we will speak about commensurate structure. The refinement of the magnetic intensities with a pure sine wave modulated structure model gives the best agreement with the neutron pattern. In this model, the Ce magnetic moments are collinear, lying along the $c$-axis. Nevertheless, within this hypothesis of a single-k sine wave magnetic structure, one small magnetic peak at $2 \theta \approx 35$ c cannot be indexed (inset of figure 2). In return, it corresponds exactly to the position of a third-order harmonic $3 \mathbf{k}$ of 
the modulated structure. On the basis of a sine wave modulated structure with two propagation vectors ( $\mathbf{k}$ and $\mathbf{3 k}$ ), the refined moment value of the Fourier component of the third harmonic is very close to $1 / 3$ of that of the first harmonic value, which strongly suggests a square wave structure. The refinement at $1.5 \mathrm{~K}$ leads to a Ce total magnetic moment of $\mu_{\mathrm{Ce}}=1.71(4) \mu_{\mathrm{B}}$ by taking account both components. The peaks corresponding to other third-order harmonic or to higher order harmonic components are too weak to be seen. The phase difference $\Delta \Phi$ between the sine wave on both Ce positions of the tetragonal unit cell has been refined as $\Delta \Phi=281{ }^{\circ}$. This value leads to a magnetic structure where the sequence of the Ce moments along the a-axis is (+ - -) for the $\mathrm{Ce}(1)$ position (1/4 1/4 0.668) and (++- ) for the $\mathrm{Ce}(2)$ position (3/4 3/4 0.332). The hypothesis of this square wave modulated structure is clearly confirmed by the refinement of the neutron diffraction pattern at $1.5 \mathrm{~K}$ directly with a commensurate square wave magnetic structure: we describe this antiphase model with constant up and down Ce magnetic moments directly in a ( $3 a$ 3a 2c) magnetic cell $\left(R_{\mathrm{B} \text {-mag. }}=6.5 \%\right)$ (figure 2$)$. The magnetic moment value is thus refined to $\mu_{\mathrm{Ce}}=$ $1.68(2) \mu_{\mathrm{B}}$, which is in fair agreement with the first model. The value is slightly smaller than that expected for the $\mathrm{Ce}^{3+}$ free ion $(2.14 \mu \mathrm{B})$ : it confirms the moderate Kondo effect in the hydride [6] but the crystal field effect may also explain the partial quenching of the Ce moment. This value is coherent also with the electronic structure calculations [20]. The low temperature magnetic structure of CeRuSiH is depicted in figure 3. When the temperature increases from $1.5 \mathrm{~K}$, magnetic peak intensities decrease (figure 4$)$ and the most intense third-order harmonic peak $\left(2 \theta \approx 35^{\circ}\right)$ totally disappears at $3.1 \mathrm{~K}$. The temperature dependence of the refined Ce magnetic moment calculated with $k$ and $3 k$ components is presented in figure 5 . Moreover the value of $k_{x}$ is constant $(1 / 3)$ up to around $3 \mathrm{~K}$ where it starts to decrease (figures 4 and 6 ). These results show that, around the $T_{\mathrm{N} 2}$ magnetic transition, the square wave modulated structure becomes strictly sinusoidal (with a propagation vector $\mathbf{k}=\left(k_{x}, k_{x}, 1 / 2\right)$ ) where $k x$ shifts from the commensurate value $1 / 3$. In other words, this transition corresponds to a delock-in of the wavevector from a commensurate value correlated with a square wave to a sinusoidal modulated structure (figure 7). This transition at $3.1 \mathrm{~K}$ is evidenced by specific heat measurements because it produces a slight decrease in the curve $C p / T=f(T)[6]$. This decrease is the sign of the sinusoidal squaring up process which has already been observed for instance in $\mathrm{TbRu}_{2} \mathrm{Si}_{2}$ and $\mathrm{TbNi}_{2} \mathrm{Si}_{2}[21,22]$.

The magnetic structures of CeRuSiH are very different from the non-hydrogenated magnetic REMX (RE = Rare Earth) compounds crystallizing in the tetragonal CeFeSi-type structure ( $M=F e$, Co or $\mathrm{Ru}, \mathrm{X}=\mathrm{Si}$ or $\mathrm{Ge})$. Indeed, contrary to CeRuSiH, the magnetic structures of these REMX compounds are all commensurate with $k=(0,0,1 / 2)$ or $(0,0,0)$ as propagation vectors and there is no evolution of these propagation vectors with the temperature [23]. Moreover, these REMX compounds can be ranked according to the sign of several RE-RE exchange interactions: $J_{0}$ within the RE planes, $J_{1}$ between RE planes separated by $M$ and $X$ planes and $J_{2}$ between the nearest RE planes as described in [23]. In the known isotypic compounds, $J_{0}$ is always positive whereas $J_{2}$ and $J_{3}$ are 
either positive or negative, depending on the nature of theM transition metal. In $\mathrm{CeRuSiH}$, the magnetic structures are more complex. On account of the magnetic modulation we observe a mixing of the interactions type: two thirds of the $J_{0}$ and $J_{2}$ and one third of the $J_{1}$ interactions are negative. In fact, the magnetic structures of CeRuSiH present more common points with some of the magnetic compounds crystallizing in the tetragonal $\mathrm{ThCr}_{2} \mathrm{Si}_{2}$-type structure like $\mathrm{NdRu}_{2} \mathrm{Si}_{2}$ and $\mathrm{TbRu}_{2} \mathrm{Si}_{2}$. Their structures are both incommensurate, sine wave modulated at temperatures just below $\mathrm{TN}$ and square wave modulated at lower temperatures [21, 24, 25]. This observation can be linked to the comparison of the interatomic distances between a Ceatom and its ligands; as said previously, the distances $d_{\mathrm{Ce}-\mathrm{Ru}}$ and $d_{\mathrm{Ce}-\mathrm{Si}}$ in the $\left[\mathrm{Ce}_{4} \mathrm{Ru}_{4}\right]$ antiprisms surrounding the Si-atom in CeRuSiH are closer to those existing in $\mathrm{CeRu}_{2} \mathrm{Si}_{2}$ than to those in CeRuSi $[6,26]$. Indeed, these distances are smaller in CeRuSi and the hydrogenation of this ternary silicide induces an increase of these lengths which become similar to those in $\mathrm{CeRu}_{2} \mathrm{Si}_{2}$. More generally, the magnetic structures of the $\mathrm{REM}_{2} \mathrm{X}_{2}$ compounds seem to depend strongly on the $a / c$ ratio ( $a$ and $c$ are the lattice parameters) and consequently on the $M$ metal transition $[27,28]$. For $a / c<0.415$, a simple collinear ordering is observed as in the compounds with $\mathrm{M}=\mathrm{Rh}, \mathrm{Co}, \mathrm{Cu}$, Fe or $\mathrm{Ir}$, while for $a / c>0.415$, the compounds exhibit oscillatory magnetic structure as in the intermetallics with $\mathrm{M}=\mathrm{Os}, \mathrm{Pd}, \mathrm{Au}$ or $\mathrm{Ru}$. If we calculate the equivalent ratio in CeRuSiH ( $c$ representing here four times the thickness of the $\left[\mathrm{Ce}_{4} \mathrm{Ru}_{4}\right]$ layer) we found 0.421 , i.e. More than 0.415 . According to this study, CeRuSiH is thus situated among the compounds with an oscillatory magnetic structure which is in agreement with the hydride magnetic structure refinement. This comparison also confirms the similarities between CeRuSiH and some of the $\mathrm{REM}_{2} \mathrm{X}_{2}$ compounds. Moreover, the magnetic phase diagram of CeRuSiH has many common points with those of the substituted systems $\mathrm{CeRu}_{2}\left(\mathrm{Si}_{1-x} \mathrm{Ge}_{x}\right)_{2}$ and $\left(\mathrm{Ce}_{1-x} \mathrm{La}_{x}\right)$ $\mathrm{Ru}_{2} \mathrm{Si}_{2}[12,13]$ : they are derived from the heavy-fermion compound $\mathrm{CeRu}_{2} \mathrm{Si}_{2}$ which is non-magnetic down to $20 \mathrm{mK}$ but presents a short-range magnetic order evidenced by neutron-scattering experiments [29]. It has been shown that long-range magnetic order could be induced by substituting a small among of either La for $\mathrm{Ce}$ or $\mathrm{Ge}$ for $\mathrm{Si}$. In both cases the substitution leads to an antiferromagnetic behaviour of these systems with magnetic moments aligned along the $c$-axis. The onset of long-range magnetism is induced by the unit cell expansion produced by the larger ionic radius of $\mathrm{La}$ and $\mathrm{Ge}$ compared to $\mathrm{Ce}$ and $\mathrm{Si}$. In both cases, the alloying can be considered as an application of a negative external pressure applied to pure $\mathrm{CeRu}_{2} \mathrm{Si}_{2}$. It is interesting to notice that the same effect of a 'negative' chemical pressure induced by $\mathrm{H}$-insertion in CeRuSi also leads to an antiferromagnetic state. In the antiferromagnetic state of $\mathrm{CeRu}_{2}\left(\mathrm{Si}_{0.9} \mathrm{Ge}_{0.1}\right) 2$, the magnetic structure just below $T_{\mathrm{N}}$ is incommensurate with a wavevector $\mathbf{k}_{1}=\left(k_{1}, 0,0\right)$ : the $k_{1}=0.308$ value is close to the one observed in $\mathrm{CeRuSiH}$, that is to say that the period of the modulation along the $a$ axis is very similar in both compounds $[12,13]$. When an external field is applied on a single crystal along the $c$ direction at constant temperature, the system undergoes two first-order phase transitions and, in the intermediate region, a second propagation vector $\mathbf{k}_{2}$ is present with the value 
$\mathbf{k}_{2}=\left(k_{1}, k_{1}, 0\right)$; in this phase, the modulation along the $a$ - and the $b$-axes is nearly the same as in CeRuSiH $[12,13]$. Moreover, in $\left(\mathrm{Ce}_{0.8} \mathrm{La}_{0.2}\right) \mathrm{Ru}_{2} \mathrm{Si}_{2}$, a second transition is observed below $\mathrm{TN}$ and it corresponds to a lock-in of the phase of the modulation, which enhances the squared modulation feature of the magnetic structure [12]. This transition is very similar to the one observed in CeRuSiH at $T_{\mathrm{N} 2} \approx 3.1 \mathrm{~K}$ which corresponds to the lock-in of the incommensurate wavevector to a rational value and to the onset of a squared modulated magnetic structure.

\section{Conclusion}

The crystal structure of CeRuSiH determined by x-ray diffraction on a single crystal has been confirmed by neutron powder diffraction. It has enabled us to localize the hydrogen atoms in the $\left[\mathrm{Ce}_{4}\right]$ tetrahedra which are totally occupied. Below antiferromagnetic transition at $T_{\mathrm{N} 1}=7.5 \mathrm{~K}$, the neutron diffraction patterns have revealed a collinear magnetic structure with $\mathbf{k}=\left(k_{x} \approx 1 / 3, k_{x}\right.$, $1 / 2$ ) as propagation vector and with a Ce magnetic moment along the $c$-axis. The $k x$ value increases when the temperature decreases until the second transition at $T_{\mathrm{N} 2}$, corresponding to the sequence incommensurate $\rightarrow$ commensurate for the magnetic structure, which also becomes square wave modulated. In the intermetallics based on cerium, the magnetic properties are governed by the competition between Kondo and RKKY interactions which is influenced by the strength of the interaction $J_{\text {cf }}$ between the $4 \mathrm{f}(\mathrm{Ce})$ and the conduction electrons. $\mathrm{H}$-insertion in CeRuSi involves an increase of the unit cell volume and thus a decrease of $J_{\text {cf. }}$ It can be considered as an application of a 'negative' pressure on CeRuSi. Neutron experiments with application of high external pressure on CeRuSiH are planned to see the consequences on the magnetic structure, especially to see if the magnetic ordering can be destroyed. It would also be interesting to investigate the CeRuGeH hydride to see the result on the magnetic order of the unit cell expansion induced by replacing Si by Ge.

\section{Acknowledgments}

The authors are indebted to the Conseil R' egional d'Aquitaine for financial support; especially ST for a PhD stipend. Finally, BC thanks the European Science Foundation (ECOM-COST action P16) for financial support.

\section{References}

[1] Chevalier B, Gaudin E, Weill F and Bobet J-L 2004 Intermetallics 12437

[2] Chevalier B and Matar S F 2004 Phys. Rev. B 70174408

[3] Chevalier B, Pasturel M, Bobet J-L and Isnard O 2005 Solid State Commun. 134529

[4] Chevalier B, Matar S F, SanchezMarcos J and Rodriguez Fernandez J 2006 Physica B 378-380

[5] Chevalier B, Matar S F, M'en 'etrier M, SanchezMarcos J and Rodriguez Fernandez J 2006 J. 
CEA / ICMCB @ 2008

Phys.: Condens. Matter 186045

[6] Chevalier B, Gaudin E, Tenc'e S, Malaman B, Rodriguez Fernandez J, Andr'e G and Coqblin B 2008 Phys. Rev. B 77014414

[7] Zimmer B I, Jeitschko W, Albering J H, Glaum R and Reehuis M 1995 J. Alloys Compounds 229 238

[8] Krellner C, Kini N S, Br"uning E M, Koch K, Rosner H, Nicklas M, Baenitz M and Geibel C 2007 Phys. Rev. B 76104418

[9] Doniach S 1977 Physica B 91231

[10] Chevalier B, Pasturel M, Bobet J-L, Decourt R, Etourneau J, Isnard O, Sanchez Marcos J and Rodriguez Fernandez J 2004 J. Alloys Compounds 3834

[11] Chevalier B, Wattiaux A and Bobet J-L 2006 J. Phys.:Condens. Matter 181743

[12] Mignod J-M, Regnault L-P, Jacoud J-L, Rossat-Mignod J, Haen P and Lejay P 1991 Physica B 171 357

[13] Mignod J-M, Boutrouille Ph, Regnault L-P, Haen P and Lejay P 1991 Solid State Commun. 77 317

[14] Süllow S, McIntyre G J, Haen P, Rainford B D and Fukuhara T 2001 J. Magn. Magn. Mater. 226230179

[15] Haen P, Bioud H and Fukuhara T 1999 Physica B 259-261 85

[16] Besnus M J, Kappler J P, Lehmann P and Mayer A 1985 Solid State Commun. 55779

[17] Rodriguez-Carvajal J 1990 Satellite Mtg 15th Congr. IUCr on Powder Diffraction (Toulouse, 1990) p 127

[18] Sears V F 1992 Neutron News 326

[19] Freeman A J and Desclaux J P 1979 J. Magn. Magn. Mater. 1211

[20] Matar S F 2007 Phys. Rev. B 75104422

[21] Salgueiro da Silva M A, Sousa J B, Chevalier B, Etourneau J, Gmelin E and Schnelle W 1995 Phys. Rev. B 5212849

[22] Blanco J A, Garnier A, Gignoux D and Schmitt D 1998 J. Alloys Compounds 275-277 565

[23] Chevalier B and Malaman B 2004 Solid State Commun. 130711

[24] Garnier A, Gignoux D, Schmitt D and Shigeoka T 1998 J. Phys.: Condens. Matter 10 3919-27

[25] Chevalier B, Etourneau J, Hagenmuller P, Quetzel S and Rossat-Mignod J 1985 J. Less-Common Met. 111161

[26] Godart C, Ammarguellat C, Ravet M F, Wetta N, Levy-Clement C, Noel H and Krill G 1986 Solid State Commun. 59241

[27] Bazela W 2007 J. Alloys Compounds 442132

[28] Szytula A and Leciejewicz J 1989 Handbook on the Physics and Chemistry of Rare Earths vol 12, ed K A Gschneidner and L Eyring (Amsterdam: Elsevier Science) p 133

[29] Haen P, Flouquet J, Lapierre F, Lejay P and Remenyi G 1987 J. Low Temp. Phys. 67391 


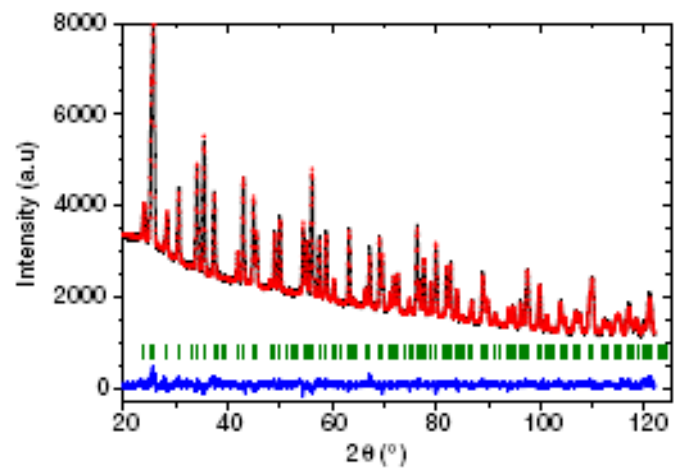

Figure 1. Rietveld profile refinement of CeRuSiH hydride at room temperature $(\lambda=1.2262 \AA)$. The full circles represent the observed data points, the solid lines reveal the calculated profile and the difference (bottom) between the observed and calculated profiles. The ticks correspond to $2 \theta$-Bragg positions.

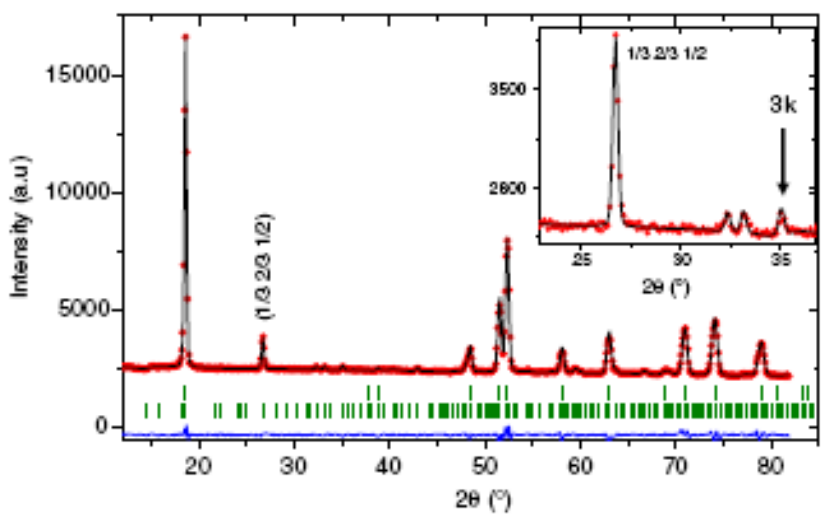

Figure 2. Observed and calculated neutron diffraction patterns of $\mathrm{CeRuSiH}$ in the square wave modulated model at $1.5 \mathrm{~K}$. The full circles represent the observed data, the continuous lines indicate the calculated and the difference (bottom) profiles. The ticks correspond to $2 \theta$-Bragg positions for the nuclear and magnetic cells. Inset: enlargement for $23^{\circ}<2 \theta<37^{\circ} ; 3 k$ arrow points out the most intense $3 k$ magnetic peak. 


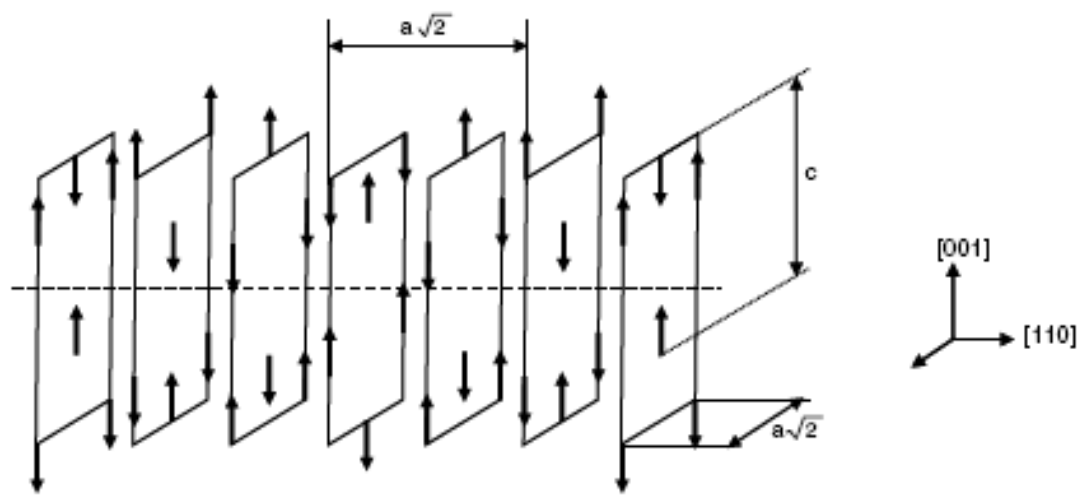

Figure 3. Schematic representation of the Ce magnetic moments alignment for the square modulated phase of CeRuSiH.

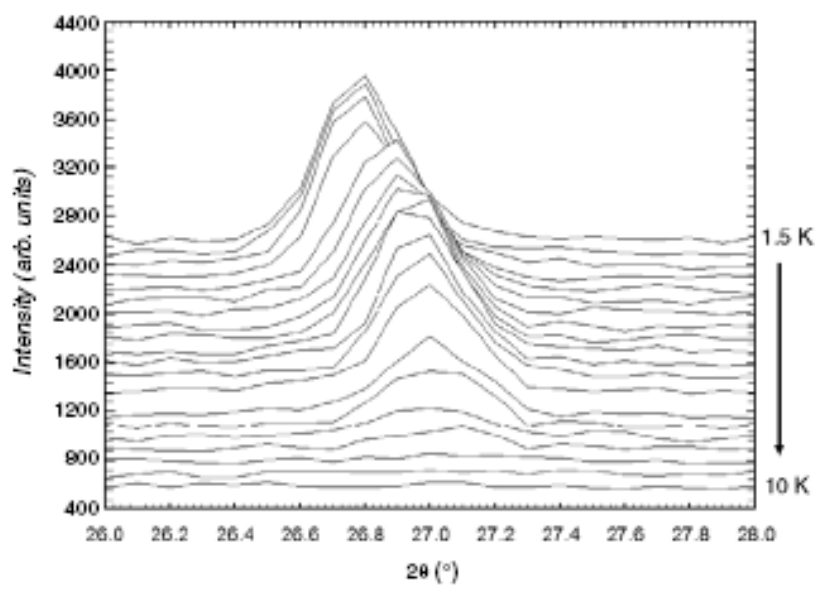

Figure 4. Temperature dependence of the most intense magnetic peak intensity for $1.5 \mathrm{~K} \leqslant T \leqslant 10 \mathrm{~K}$, showing that the position of this peak is quite fixed up to about $3 \mathrm{~K}$ and starts to move as $T$ continues to increase. The different temperatures are $1.5,2.1,2.6$, $2.8,3.1,3.3,3.5,3.7,3.9,4.0,4.5,5.0,5.5,6.0,6.5,7.0,7.3,7.6,7.9$ and $10 \mathrm{~K}$.

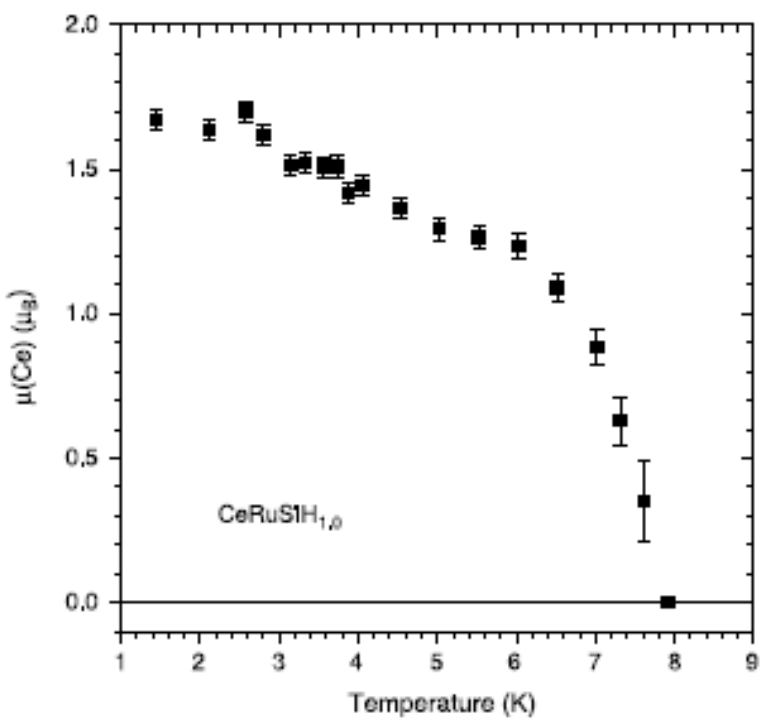

Figure 5. Temperature dependence of the Ce magnetic moment in the CeRuSiH hydride. 


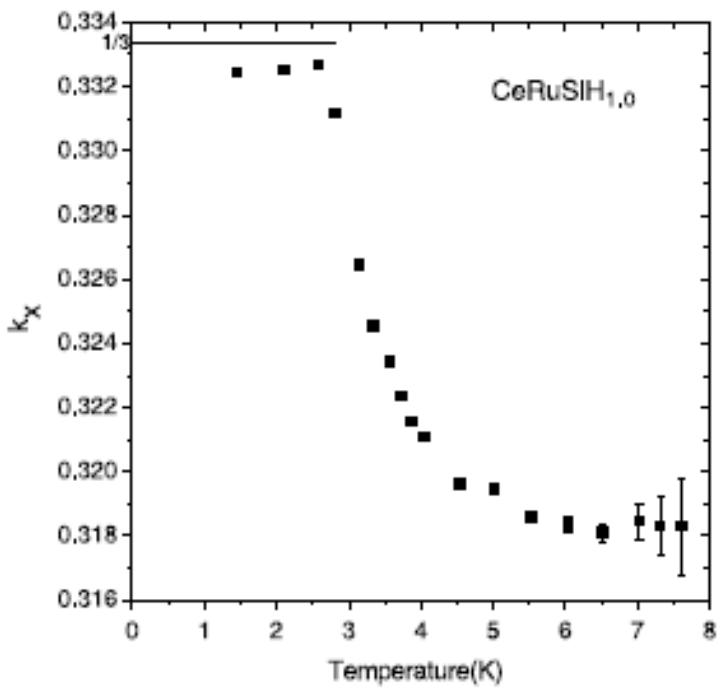

Figure 6. Temperature dependence of the $k_{x}$ component of the $\mathbf{k}=\left(k_{x}, k_{x}, 1 / 2\right)$ propagation vector associated to the magnetic structures of CeRuSiH.

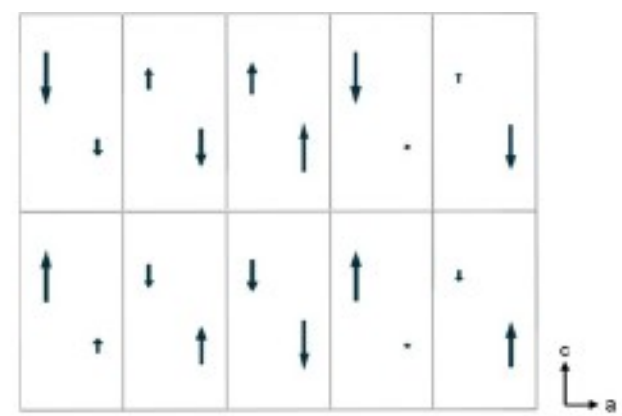

Figure 7. Projection onto the $(a, c)$-plane of the sine wave magnetic structure of $\mathrm{CeRuSiH}$ at $4 \mathrm{~K}$. Only the $\mathrm{Ce}$ magnetic moments are presented and the rectangles delimit the nuclear cells.

Table 1. Positional and isotropic thermal parameters at room temperature for $\mathrm{CeRuSiH}$ ( $3 \mathrm{~T} 2$ data). The reliability factors are $R_{\text {wp }}=8.7 \%$ and $R_{\mathrm{B}}=4.9 \%$, respectively.

\begin{tabular}{|c|c|c|c|c|c|}
\hline \multirow[b]{2}{*}{ Atom } & \multirow[b]{2}{*}{ Site } & \multicolumn{3}{|c|}{ Positional parameters } & \multirow[b]{2}{*}{$B_{\operatorname{ISO}}\left(\AA^{2}\right)$} \\
\hline & & $X$ & $y$ & $z$ & \\
\hline $\mathrm{Ce}$ & $2 c$ & $1 / 4$ & $1 / 4$ & $0.6680(2)$ & $0.64(2)$ \\
\hline $\mathrm{Ru}$ & $2 a$ & $3 / 4$ & $1 / 4$ & 0 & $0.46(2)$ \\
\hline $\mathrm{Si}$ & $2 c$ & $1 / 4$ & $1 / 4$ & $0.1495(2)$ & $0.49(2)$ \\
\hline $\mathrm{H}$ & $2 b$ & $1 / 4$ & $3 / 4$ & $1 / 2$ & $2.04(5)$ \\
\hline
\end{tabular}

